\title{
MED16/SFR6 IS NECESSARY BUT NOT SUFFICIENT FOR COR GENE EX- PRESSION OF CBF PATHWAY
}

\author{
Deepthi Lakmini Wathugala ${ }^{1 *}$, Heather Knight ${ }^{2}$ and Marc R Knight ${ }^{2}$ \\ Department of Crop Science, Faculty of Agriculture, University of Ruhuna, Mapalana, Kamburupitiya, Sri \\ Lanka ${ }^{2}$ School of Biological and Biomedical Sciences, Durham University, United Kingdom
}

Accepted: $5^{\text {th }}$ November 2011

\begin{abstract}
Developing stress tolerant crops to cope with the rapid environmental degradation that is occurring is an absolute requirement in order to provide enough food for growing population. One of the basic genetic engineering approaches currently being used to improve crop stress tolerance is generation of transgenic plants by introducing novel genes into the genome of agriculturally important crops or altering the expression of existing genes. Understanding stress response signaling pathways is the prime requirement to manipulate stress tolerance of crop plants by this approach. SFR6 (SENSITIVE TO FREEZING6) is one of plant mediator protein which has identified first with its involvement to tolerance against freezing in Arabidopsis. The freezing sensitivity of SFR6 mutant is lack of expression of downstream genes in CBF cold response pathway. SFR6 also mediates tolerance to osmotic stress induced by drought and salinity. However, the over expression of SFR6 in wild type Arabidopsis did not increase COR (Cold on regulated) gene expression under ambient temperature. Therefore, the effect of SFR6 on cold gene expression was further characterized in this study. Results showed that over expression of SFR6 did not alter $C B F 1$ expression levels under ambient temperature as well as $C O R$ gene expression under cold and osmotic stress indicating SFR6 alone cannot use as a molecular tool to improve stress tolerance of crop plants. Further over expression of both SFR6 and $C B F 1$ also did not have additive effect on $C O R$ gene expression. Therefore, further studies of the mechanism of SFR6 on $C O R$ gene regulation need to be conducted to evaluate the potential of use of SFR6 as a molecular tool to increase stress tolerance of crop plants.
\end{abstract}

Key words: CBF Pathway, COR Gene, MED16/ SFR6, Stress Tolerance

\section{INTRODUCTION}

Low temperature is an adverse environmental condition that affects plant growth and development and thus crop yield reduce mainly in temperate countries and higher altitudes in tropical countries. Cold acclimation is important in mediating the freezing tolerance of plants grown in these regions. During the acclimation process numerous molecular and physiological changes take place including alterations in gene expression. The complexity of this process is implied by the number of genes altered during cold acclimation. According to some estimations $25 \%$ of the transcriptome in Arabidopsis is involved in cold acclimation (Kreps et al. 2002). Many are also inducible by drought (Thomashow 1999). Therefore, a basic understanding of the molecular mechanisms of cold acclimation-related gene expression and the signaling pathways leading to them is important to improve the freezing and drought tolerance of agricultural crops.

\footnotetext{
*Corresponding author: wgdlakmini@crop.ruh.ac.lk
}

A group of transcription factors called C-repeat binding factors (CBF) (Stockinger et al. 1997), and also known as dehydration responsive element binding factors (DREB1) (Liu et al. 1998), control cold induced COR (Cold on regulated) gene expression. $\mathrm{CBF} / \mathrm{DREB} 1$ transcription factors belong to the EREBP/AP2 family of DNA binding proteins (Stockinger et al. 1997; Liu et al. 1998). There are 3 closely related cold inducible $C B F / D R E B 1$ genes, named $C B F 1 / D R E B 1 B, C B F 2 / D R E B 1 C$ and $C B F 3 / D R E B 1 A$ all located on chromosome 4 of Arabidopsis with approximately $88 \%$ identical and $91 \%$ similar amino acid sequences (Gilmour et al. 1998; Liu et al. 1998). CBF/ DREB1 transcripts increase markedly within 15 minutes of transfer to low temperature, and continue to increase over next 1-2 h. These 3 genes encode transcriptional activators which bind CRT/DRE elements (Gilmour et al. 1998; Liu et al. 1998). After about $2 \mathrm{~h}$, the transcripts of COR genes containing CRT/DRE in the promoters accumulate (Gilmour et al. 1998). The 
over-expression of $C B F / D R E B 1$ transcription factors in Arabidopsis result in a great increase in freezing tolerance in wild type Arabidopsis (Jaglo-Ottosen et al. 1998; Liu et al. 1998). AtSFR6 a plant mediator protein (Backstrom et al. 2007) has also been identified as a protein that regulate $\mathrm{CBF} / \mathrm{DREB}$ dependent $C O R$ gene expression in Arabidopsis (Knight et al. 2009). The freezing sensitivity of $s$ fr 6 mutants are due to defective expression of COR genes controlled by $\mathrm{CBF} / \mathrm{DREB} 1$ transcription factor (Knight et al. 1999; Knight et al. 2009). However, $C B F / D R E B 1$ gene expression is not misregulated at the transcriptional and translational level in sfr6, indicating that SFR6 operates downstream of CBF translation (Knight et al. 1999; Knight et al. 2009). However, over expression of AtSFR6 did not increase KIN2 expression in wild type Arabidopsis (Wathugala et al. 2011). SFR6 also mediates tolerance to osmotic stress induced by drought and salinity (Knight et al. 1999; Boyce et al. 2003) and gene expression affecting other important developmental processes such as flowering (Knight et al. 2008). AtSFR6 homologue has also been identified in rice and over expression of OSSFR6 in Arabidopsis sfr6-1 mutant showed functional orthology through genetic complementation (Wathugala et al. 2011). Therefore, the effect of SFR6 on cold gene expression was further characterized in this study.

\section{MATERIALS AND ME-THODS}

\section{Production of both AtSFR6 and AtCBF1 over expressing lines}

Crosses between $35 S:: A t C B F 1$ plants in Col-0 background (Knight et al. 2009) with 35S::AtSFR6 in sfr6-1 mutant background (Wathugala et al. 2011) were performed to construct Arabidopsis lines over-expressing both CBF1 and SFR6 genes. 35S::AtSFR6 plants were used as recipient plants when crossing. Col-0 and sfr6-1 mutant plants were also crossed with $35 S:: A t C B F 1$ as controls. As $35 S:$ AtCBF 1 plants harbour a kanamycin (Kan) resistant gene F1 plants were selected on Kan supplemented MS agar plates. Plants were grown to maturity in a growth room maintained at approximately $20 \pm 1{ }^{\circ} \mathrm{C}$ with a long day photoperiod ( $16 \mathrm{~h}$ light $/ 8 \mathrm{~h}$ dark) and light level of $100-150 \mu \mathrm{mol} \mathrm{m} \mathrm{m}^{-2} \mathrm{~s}^{-1}$ and F2 seeds from individual plants collected. As 35S::AtSFR6 construct harbour a phosphinothricine $(P P T)$ resis- tant gene F2 seeds were germinated on MS agar plates supplemented with Kan and $P P T$. The seeds from Col-0 and sfr6-1 crossed with $35 S:: A t C B F 1$ were selected on Kan supplemented agar plates. Twelve day old plants were harvested for RNA extraction. sfro- 1 mutants have pale green colored leaves (Knight et al., 2009). Therefore, seedlings with pale coloured leaves on Kan supplemented plates were selected as homozygous sfr6-1 over-expressing $C B F 1$.

\section{Cold stress}

Seven-day-old seedlings grown on horizontal agar plates were transferred to $4^{\circ} \mathrm{C}$ for the appropriate amount of time in constant light. The whole plants were collected after the indicated time of cold exposure and quickly frozen in liquid nitrogen. One biological replicate contained $15-20$ seedlings.

\section{Osmotic stress}

Seven-day-old seedlings grown on MS agar plates were floated in $6 \mathrm{ml}$ of sterile water or $350 \mathrm{mM}$ mannitol contained in transparent multi well plates. Before adding mannitol, seedlings were floated in $3 \mathrm{ml}$ of sterile water for $3 \mathrm{~h}$ to recover from transfer to water from agar medium. Then, $3 \mathrm{ml}$ of $700 \mathrm{mM}$ mannitol solution was added to each well, except for control plants to which $3 \mathrm{ml}$ of water was added. Fifteen to 20 seedlings were used for each treatment. Samples were collected $6 \mathrm{~h}$ post treatment. Immediately before harvesting, seedlings were blotted on tissue paper to remove excess solution. Samples were then quickly placed into microfuge tubes and snapfrozen in liquid nitrogen. Samples were processed so that the minimum time elapsed (less than 1 minute) between harvesting and freezing.

\section{Quantitative real time PCR}

A high capacity cDNA reverse transcription kit (Applied Biosystems, Foster City, CA, USA) was used to reverse transcribe cDNA from $1.5 \mu \mathrm{g}$ total RNA extracted using the Quagen Rneasy plant mini kit in conjunction with RNAse-free DNase (Quagen) to remove any genomic DNA contamination. Quantitative real time PCR (qRT-PCR) was performed on $10 \mu 1$ of 1 : 50 diluted cDNA reaction in a $25 \mu 1$ reaction using an Applied Biosystem 7300 system. Relative transcript abundance of KIN2 
(At5g15970; At02354775_s1) were measured using gene-specific TaqMan probes from Applied Biosystem.

\section{RESULTS}

SFR6 gene controls freezing and osmotic stress tolerance in Arabidopsis (Knight et al. 1999, 2009; Boyce et al. 2003). AtSFR6 has been identified as At4g04929 (Knight et al. 2009) a plant mediator sub-unit (Backstrom et al. 2007). Overexpression of AtSFR6 in sfr6-1 mutant background complemented all visible phenotypes of sfr6-1 mutant (Wathugala et al. 2011). However, overexpression of AtSFR6 in wild type Arabidopsis did not alter downstream cold gene expression (Wathugala et al. 2011) indicating that SFR6 alone might not influence activation of target COR genes in Arabidopsis. Over-expression of $C B F$ genes in the sfr6-1

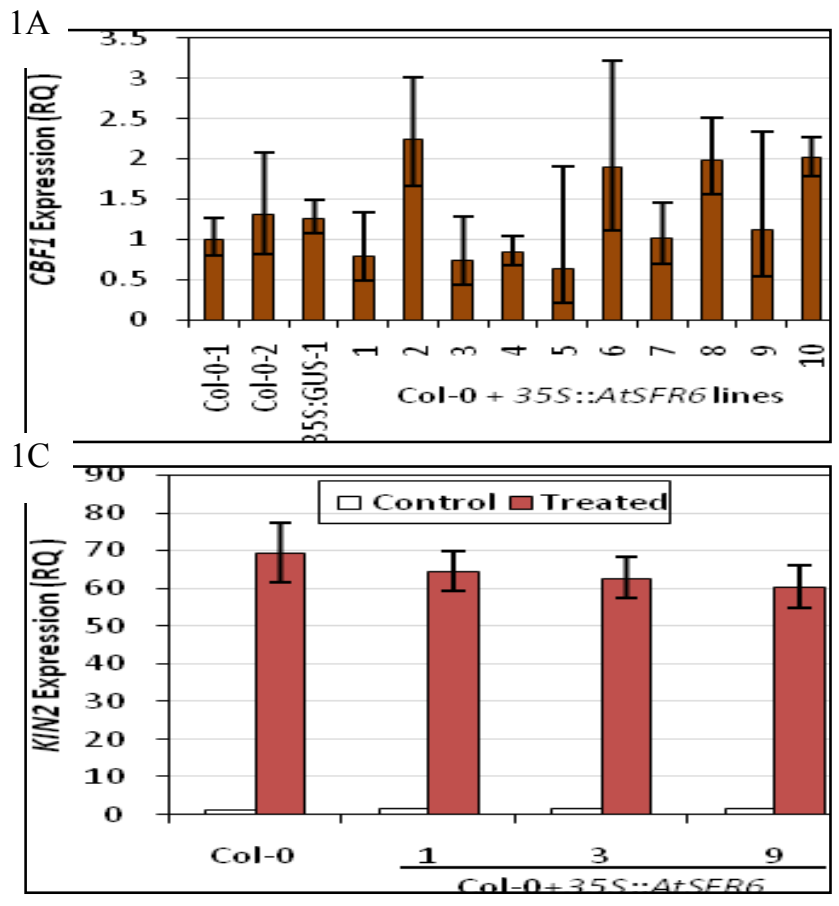

mutant did not alter COR gene expression (Knight et al. 2009). However, over-expression of $C B F$ transcription factors in wild type Arabidopsis causes large increase in COR gene expression and freezing tolerance at ambient temperatures (Jaglo-Ottosen et al. 1998; Liu et al. 1998; Kasuga et al. 1999; Gilmour et al. 2000). Therefore, the effect of overabundance of AtSFR6 on $C B F 1$ transcript levels was first analysed.

The same cDNA samples prepared to determine KIN2 and SFR6 levels (Wathugala et al. 2011) were used. Apart from 4 lines all other lines showed comparatively reduced levels of $C B F 1$ expression (Fig 1A), but there is no significant difference. Although the overabundance of AtSFR6 has no effect on KIN2 (Wathugala et al. 2011) and CBF1 expression in unstressed seedlings, there is, however, a
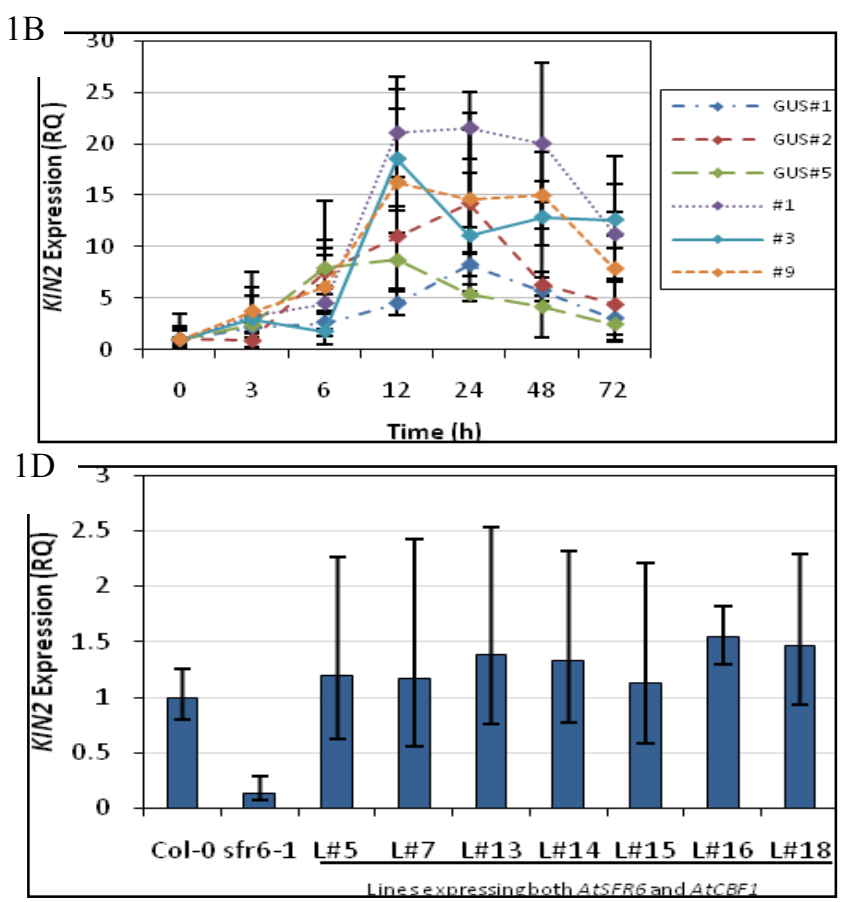

Figure 1: (A)Expression of $C B F 1$ in unstressed 35S::AtSFR6 over-expressing lines. Graph shows the relative quantification (RQ) values for $C B F 1$ expression relative to the level of $C B F 1$ expression in non-transgenic Col- 0 plants. $\beta$ TUBULIN4 was used as endogenous control. (B) KIN2 expression during a time course of cold exposure in Col+35S:: AtSFR6 lines and Col+35S::GUS lines. Graph shows relative quantification (RQ) values of KIN2 levels relative to the KIN2 level of each line's unstressed conditions. $\beta$-TUBULIN4 was used as an endogenous control.

(C) KIN2 expression after osmotic stress in Col-0+35S:: AtSFR6 lines. Relative quantification (RQ) of KIN2 expression relative to the KIN2 level of non-transgenic Col-0 treated with water are shown here. $\beta$-TUBULIN4 used as endogenous control.

(D) Transcript levels of KIN2 in transgenic lines expressing both 35S::AtSFR6 and 35S::AtCBF1. Data represented here are relative quantification (s) values of KIN2 expression relative to the KIN2 levels of Col-0 over-expressing CBF1. PEX4 was used as endogenous control.

Each value is the mean of three technical replicates. Error bars indicate $R_{\mathrm{MIN}}$ and $\mathrm{RQ}_{\mathrm{MAX}}$ and constitute the acceptable error for a $95 \%$ confidence limit according to Student's $t$ test. 
possibility that SFR6 has its effect after low temperature stimuli. To test this, the expression of KIN2 was further characterized in SFR6 over-expressing transgenic lines during a time course of cold exposure. Three Col- $0+$ $35 S:: G U S$ lines were used as controls and three Col-0 + 35S::AtSFR6 transgenic lines (Line \#1, \#3 and \#9) (Wathugala et al. 2011) with different levels of SFR6 expression (strong, moderate and weak respectively) were chosen.

KIN2 transcripts increased up to a maximum in $12 \mathrm{~h}$ cold treatments and then gradually decreased (fig. 1B). The three SFR6 overexpressing transgenic lines showed higher KIN2 transcript level than three GUS lines from 12 to $72 \mathrm{~h}$ (fig.1B). At the end of 3days $(72 \mathrm{~h})$ of $4^{\circ} \mathrm{C}$ exposure transcript levels of KIN2 in GUS lines had returned to ambient levels. Apart from these changes no consistent difference in expression of KIN2 was observed in any time course between the AtSFR6 overexpression lines and the $G U S$ expression lines. These results suggest that SFR6 may have effect to keep KIN2 expression at a higher level for a longer period of time.

The expression of KIN2 was characterized further by subjecting plants to osmotic stress, as the $s f r 6-1$ mutant displayed reduced expression of $C O R$ genes after mannitol induced osmotic stress (Boyce et al. 2003). However, there was no significant difference between nontransgenic Col-0 and 35S::AtSFR6 overexpressers (fig. 1C) further suggesting that the over-expression of SFR6 alone does not have an effect on KIN2 expression.

Although we have evidence that SFR6 is essential for $C O R$ gene expression of $C B F$ cold response pathway, all the above analyses suggested that the over-expression of SFR6 alone is not sufficient to induce cold gene expression. However, the over expression of $C B F 1$ alone significantly increases cold tolerance in Arabidopsis (Jaglo-Ottosen et al. 1998) by increasing levels of COR gene expression. As SFR6 does not have effect on $C B F$ gene expression, the additive effect of $C B F 1$ and SFR6 overexpression on KIN2 expression was determined.

Crosses between $35 S:: A t C B F 1$ plants in Col-0 background (Knight et al. 2009) with
35S::AtSFR6 in sfr6-1 mutant plants (Wathugala et al. 2011) were performed to construct Arabidopsis lines over-expressing both $C B F 1$ and SFR6 genes. A slight increase of KIN2 could be observed in transgenic lines over-expressing both $S F R 6$ and $C B F 1$ but these differences were not significant (95\% confidence limit according to Student's $t$ test) (Fig. 1D).

\section{DISCUSSION}

Previous work identified $A t 4 g 04920$ a plant mediator gene as the locus for SFR6; a gene required for $C O R$ gene expression and cold acclimation in Arabidopsis (Knight et al. 2009). However the overexpression of AtSFR6 in wild type Arabidopsis did not increase KIN2 expression either in ambient temperatures (Wathugala et al. 2011) or after cold exposure indicating that the levels of SFR6 alone might not influence activation of target $C O R$ genes in Arabidopsis.

Previous studies also showed reduced $C O R$ gene expression in the sfr6-1 mutant, not only after cold exposure, but also after osmotic stress induced by mannitol (Boyce et al. 2003). However, KIN2 expression in AtSFR6 overexpressing lines after osmotic stress did not alter compared to wild type Arabidopsis. These results further confirm SFR6 is necessary but not sufficient for $C O R$ gene expression, not only at low temperature, but also in response to osmotic stress.

Over-expression of $C B F$ genes in the sfro-1 mutant did not alter $C O R$ gene expression (Knight et al. 2009). However, over-expression of $C B F$ transcription factors in wild type Arabidopsis causes large increase in COR gene expression and freezing tolerance at ambient temperatures (Jaglo-Ottosen et al. 1998; Liu et al. 1998; Kasuga et al. 1999; Gilmour et al., 2000). Therefore, the possible additive effect on $C O R$ gene expression due to an overabundance of SFR6 and $C B F$ was examined. The data showed that increasing both SFR6 and $C B F 1$ had no effect on $C O R$ gene expression indicating that overabundance of both SFR6 and $C B F 1$ has no additive effect on $C O R$ gene expression.

As At4g04920 is a sub unit of plant mediator 
complex (Backstrom et al. 2007) one explanation for the lack of effect when SFR6 is overexpressed in wild type is that the SFR6 protein is required in stoichiometric proportions with one or more other proteins as a part of a complex and that an increase in the amount of any one of these individually cannot influence the amount of the complex as a whole.

Mediator is the evolutionary conserved multiprotein complex that binds RNA polymerase II and controls transcription of genes (Flanagan et al. 1991). The possible mechanism suggested by Chadick and Asturias (2005), Bjorklund and Gustafsson (2005), and Haha (2004) for transcriptional activation by mediator was that the gene specific activators (transcription factors) recruits mediator to the transcription initiation site. Then, general transcriptions factors involved in gene transcription interact with the DNA-mediator complex to form a platform to bind RNA polymerase II. According to this mechanism when plants are exposed to low temperature $\mathrm{CBF}$ proteins activate and bind to the promoter of $C O R$ genes and recruits mediator complex to the promoter to build the RNA polymerase assembly platform. It is possible that MED16/SFR6 might directly bind with CBF or with other mediator subunit which links with MED16 (Myers et al. 1999; Chadick and Asturias, 2005). Based on these results over-expression of a single gene of the mediator complex does not result in increased activity of the complex as a whole.

\section{CONCLUSION}

In conclusion the results reported here showed that there is no possibility to use of AtSFR6 alone as a molecular tool to improve crop tolerance to environmental stress. As well as there is no additive effect on $C O R$ gene expression by over expressing both AtSFR6 and AtCBF1. Therefore, the mechanism of regulation of stress induced gene expression via SFR6/ MED16 remains to be further investigated. The future research on specific roles of individual subunits and of the whole complex will widen our knowledge of the transcriptional regulation of gene expression in plant and will create new routes to improve crop tolerance to environmental stress.

\section{REFERENCES}

Backstrom S, Elfving N, Nilsson R, Wingsle G, Bjorklund S 2007 Purification of a plant mediator from Arabidopsis thaliana identifies PFT1 as the Med25 subunit. Molecular Cell 26: 717-729

Bjorklund S, Gustafsson CM 2005 The yeast Mediator complex and its regulation. Trends in Biochemical Sciences 30: 240-244

Boyce JM, Knight H, Deyholos M, Openshaw MR, Galbraith DW, Warren G, Knight MR 2003 The sfr6 mutant of Arabidopsis is defective in transcriptional activation via CBF/DREB1 and DREB2 and shows sensitivity to osmotic stress. Plant Journal 34: 395-406

Chadick JZ, Asturias FJ 2005 Structure of eukaryotic Mediator complexes. Trends in Biochemical Sciences 30: 264-271

Flanagan PM, Kelleher RJ 11l, Sayre MH, Tschochner $\mathrm{H}$ and Kornberg RD 1991 A mediator required for activation of RNA polymerase 11 transcription in vitro. Nature 350: 436-438

Gilmour SJ, Sebolt AM, Salazar MP, Everard JD, Thomashow MF 200) Overexpression of the Arabidopsis $\mathrm{CBF} 3$ transcriptional activator mimics multiple biochemical changes associated with cold acclimation. Plant Physiology 124: 1854-1865

Gilmour SJ, Zarka DG, Stockinger EJ, Salazar MP, Houghton JM, Thomashow MF 1998 Low temperature regulation of the Arabidopsis CBF family of AP2 transcriptional activators as an early step in cold-induced COR gene expression. Plant Journal 16: 433-442

Hahn S 2004 Structure and mechanism of the RNA polymerase II transcription machinery. Nature Structural \& Molecular Biology 11: 394-403

Jaglo-Ottosen KR, Gilmour SJ, Zarka DG, Schabenberger O, Thomashow MF 1998 Arabidopsis CBF1 overexpression induces COR genes and enhances freezing tolerance. Science 280: 104106

Kasuga M, Liu Q, Miura S, Yamaguchi-Shinozaki K, Shinozaki K 1999 Improving plant drought, salt, and freezing tolerance by gene transfer of a single stress-inducible transcription factor. Nature Biotechnology 17: 287-291

Knight H, Mugford SG, Ulker B, Gao DH, Thorlby G, Knight MR 2009 Identification of SFR6, a key component in cold acclimation acting posttranslationally on CBF function. Plant Journal 58: 97-108

Knight H, Thomson AJW, McWatters HG 2008 Sensitive to freezing6 integrates cellular and environmental inputs to the plant circadian clock. Plant Physiology 148: 293-303

Knight H, Veale EL, Warren GJ, Knight MR 1999 The sfr6 mutation in Arabidopsis suppresses low 
-temperature induction of genes dependent on the CRT DRE sequence motif. Plant Cell 11: 875-886

Kreps JA, Wu YJ, Chang HS, Zhu T, Wang X, Harper JF 2002 Transcriptome changes for Arabidopsis in response to salt, osmotic, and cold stress. Plant Physiology 130: 2129-2141

Liu Q, Kasuga M, Sakuma Y, Abe H, Miura S, Yamaguchi-Shinozaki K, Shinozaki K 1998 Two transcription factors, DREB1 and DREB2, with an EREBP/AP2 DNA binding domain separate two cellular signal transduction pathways in drought- and low-temperature-responsive gene expression, respectively, in Arabidopsis. Plant Cell 10: 1391-1406

Stockinger EJ, Gilmour SJ, Thomashow MF 1997 Arabidopsis thaliana CBF1 encodes an AP2 domain-containing transcriptional activator that binds to the C-repeat/DRE, a cis-acting DNA regulatory element that stimulates transcription in response to low temperature and water deficit. Proceedings of the National Academy of Sciences of the United States of America 94: 10351040
Thomashow MF 1999 Plant cold acclimation: Freezing tolerance genes and regulatory mechanisms. Annual Review of Plant Physiology and Plant Molecular Biology 50: 571-599

Wathugala DL, Shane A. Richards, Heather Knight, Marc R. Knight 2011 OsSFR6 is a functional rice orthologue of SENSITIVE TO FREEZING-6 and can act as a regulator of $C O R$ gene expression, osmotic stress and freezing tolerance in Arabidopsis, New Phytologist. 191 (4): 984-995 